\title{
Proteome analysis of the bovine milk fat globule: enhancement in the membrane purification
}

\author{
C. Vanderghem¹, C. Blecker ${ }^{1}$, S. Danthine' ${ }^{1}$ C. Deroanne ${ }^{1}$, E. Haubruge ${ }^{2}$, E. De Pauw ${ }^{3}$, F. Francis \\ 1 Technologie des Industries Agro-alimentaires, Gembloux Agricultural University, Belgium \\ 2 Functional and Evolutionary Entomology, Gembloux Agricultural University, Belgium \\ 3 Mass Spectrometry Laboratory, University of Liège, Belgium \\ vanderghem.c@fsagx.ac.be
}

\section{Introduction}

The MFGM is the protective coat surrounding lipid globules in milk, which allows the dispersion of the fat in the milk plasma. This coat material is a tripartite structure that is partially derived from the apical plasma membrane, from the endoplasmic reticulum and from other intracellular compartments of the mammary epithelial cell. This membrane prevents flocculation and coalescence of lipid droplets and protects the fat against lipolysis (1). As MFGM acts as a natural emulsifying agent, it should be considered as a potential stabilizing agent in the preparation of certain foods, e.g. creams and emulsions, infant formulas, and reduced-fat products (2). Furthermore, in recent years, different factors with health beneficial properties (cholesterolemia-lowering factor, inhibitions of cancer cell growth...) have been detected in bovine MFGM components (3).

The protein composition of MFGM is completely different from the skim milk one. Milk is composed of numerous specific proteins (caseins, whey proteins). Even if bovine skim milk proteins are well identified and characterized, the total identification of the bovine MFGM proteins has not yet been concluded. Many proteins that are present in low concentration may be easily missed due to the overwhelming amounts of other contaminant proteins. Therefore, it is often necessary to reduce the complexity of the mixtures before the proteins can be identified

The application of a method to obtain purified fat globules with a minor non globular-component is detailed. Additionally, four different detergents were tested: CHAPS, amidosulfobetaine-14 (ASB 14), Sodium Lauroyl sarcosinate (Sarkosyl) and Sodium deoxycholate, in order to maximize the membrane proteome extraction to be observed by following 2-DE.

MALDI-TOF MS and MS/MS was used to identify most proteins from the MFGM

Methodology

Preparation of the milk fat globule membrane

The MFGM was extracted from fresh unpasteurised cream (Holstein milk). Cream was washed with phosphate buffer $(0.01 \mathrm{M}$ Na2HPO4/NaH2PO4; 0.9\% NaCl; pH 7.2$)$ and subjected to centrifugations between washes. Washed fat clobules were resuspended in distilled water and allowed to crystallise for twenty hours as $4^{\circ} \mathrm{C}$ Globules were burned $14^{\circ} \mathrm{C}$ until fot and sera (MFGM suspension) were separated. The total sera was centrifuged twice ( $5000 \mathrm{x} g .15$ $\left.\min , 4^{\circ} \mathrm{C}\right)$ to remove the fat and freeze dried

Sample preparation optimization for analysis of membrane proteins by 2-DE

In order to improve extraction and solubilization of the hydrophobic membrane proteins four detergents at different concentrations were tested in the sample preparation. Membrane proteins were precipitated by the addition of TCA. The pellet was then solubilized in rehydratation buffer before proteins separation by IEF and SDS-PAGE electrophoresis.

Protein spots of interest from bovine MFGM were excised from 2-DE, trypsin digested and identified by PMF and subsequent MS/MS analysis

\section{Results}

1. Extraction of membrane proteins by detergents

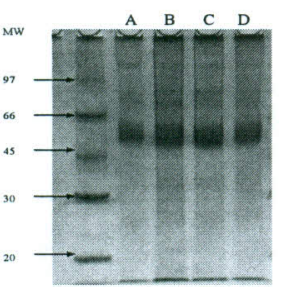

Fig1. 1D SDS-PAGE pattern of MFGM extracted with A) $0,5 \%$ Sodium Lauroyl sarcosinate, B)
ASB 14, D) $0,5 \%$ Sodium deoxycholate

The protein bands observed in the patterns for the samples of all detergents showed a similar sequence migration of the protein However, zwitteronic detergents (CHAPS and ASB 14) allowed to extract the largest quantity of membrane proteins. The polypeptide pattern for MFGM proteins show three major bands that could correspond to lactadherin, butyrophilin and adipophilin. As there was no improved banding patterns over the four detergents,
subsequent analysis by 2-DE were performed with the four detergents.

Samples extracted with zwitteronic detergents showed improvemen over the spots patterns. No significant difference was observe interest were soluble under electrophoresis conditions.
2. 2-DE analysis of milk fat globule membrane

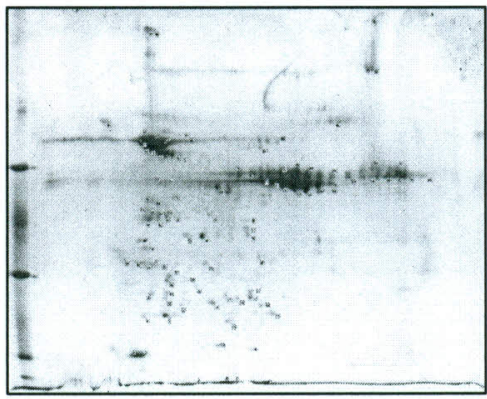

Fig 2. 2-DE of MFGM extracted with $4 \%$ CHAPS. Preparative gel was stained with Coomassie blue.

Milk fat globule proteins counts for $2-4 \%$ of the total milk protein content. Abundant proteins may mask identification of less abundant proteins of interest. In the 2-DE no spots were detected in the typical area of non-MFGM proteins. Our preparation of the MFGM allowed optimal fracion

The large majority of the MFGM proteins appeared at a $\mathrm{p} /$ between 4 and 8 Dat files from Maldi analysis were searched against Mammalian protein database.

found, suggesting post-translational proteins. A total of 79 spots matched bovine proteins. The other 3 spots were
matched to homologous proteins in human (spot 5 and 6 ) and rabbit (spot 18)
3. Identifications of proteins by PMF and subsequent MS/MS

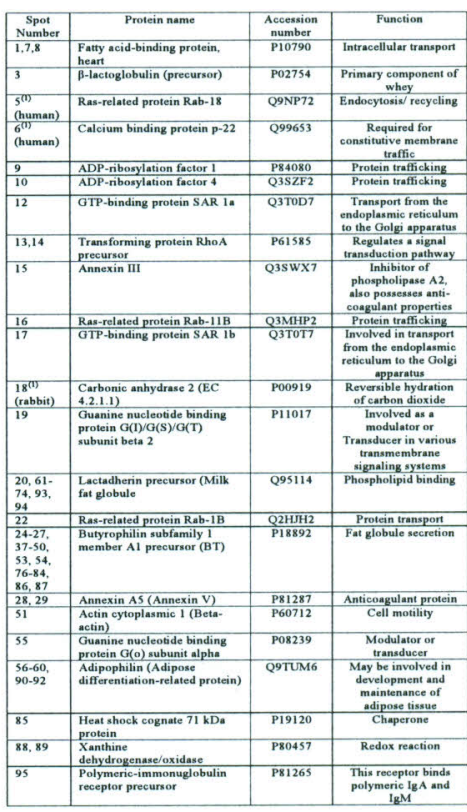

(1) Protein matched to homologous

\section{Conclusions}

Our method for the MFGM preparation allowed maximal elimination of non-MFGM protein

- Extraction of membrane proteins by $4 \%$ CHAPS or $4 \%$ ASB 14 gave good results on a 2-DE map

- Presence of the most abundant proteins in the MFGM and further identification of MFGM minor proteins for additional completion of the proteome were confirmed

References 1 .Danthine, S., Blecker, C., Paquol, M., Innocente, N., Deroanne, C. 2000. Evolution des connaissances sur la membrane du globule gras du lait: synthèse bibliographique. Lait 80:209-222

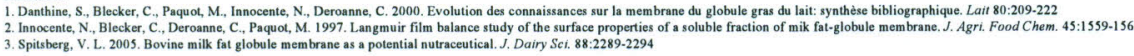

